\title{
Understanding noninvasive charge transfer doping of graphene: a comparative study
}

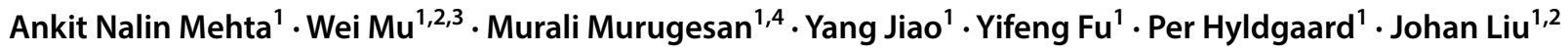

Received: 21 July 2017 / Accepted: 18 December 2017 / Published online: 8 February 2018

(c) The Author(s) 2018. This article is an open access publication

\begin{abstract}
In this work, we systematically investigate and compare noninvasive doping of chemical vapor deposition graphene with three molecule dopants through spectroscopy and electrical conductivity techniques. Thionyl chloride shows the smallest improvement in conductivity with poor temporal and thermal stability and nitric acid induces the biggest sheet resistance reduction with modified stability. Molybdenum trioxide doping stands out, after thermal annealing, with both causing a significant sheet-resistance reduction and having superior temporal and thermal stability. These properties make it ideal for applications in advanced electronics. Theoretical studies based on the van der Waals density functional method suggest that cluster formation of molybdenum trioxide underpins the significant reduction in sheet resistance, and the stability, that arises after thermal annealing. Our comparative study clarifies charge transfer doping of graphene and brings understanding of the weak-interaction nature of such non-destructive doping of graphene. Our work also shows that we can use weak chemisorption to tailor the electronic properties of graphene, for example, to improve conductivity. This ability open up possibilities for further use of graphene in electronic interconnects, field effect transistors and other systems.
\end{abstract}

\section{Introduction}

Graphene is attractive due to its very high carrier mobility and current-carrying capacity. It is ideal for applications as transparent conductors, electronic interconnects, field effect transistors and more [1]. Graphene doping is being investigated as a viable technique to tailor its electronic properties

Electronic supplementary material The online version of this article (https://doi.org/10.1007/s10854-017-8443-8) contains supplementary material, which is available to authorized users.

Murali Murugesan

murali.murugesan@chalmers.se

$\triangle$ Johan Liu

johan.liu@chalmers.se

1 Electronics Materials and Systems Laboratory, Department of Microtechnology and Nanoscience (MC2), Chalmers University of Technology, Kemivägen 9, 41296 Göteborg, Sweden

2 SMIT Center and School of Mechatronics and Mechanical Engineering, Shanghai University, No 20, Chengzhong Road, Shanghai, China

3 Fingerprint Cards AB, Box 2412, 40316 Göteborg, Sweden

4 Present Address: SHT Smart High-Tech AB, Aschebergsgatan 46, 41133 Göteborg, Sweden and improve conductivity. Doping of graphene through functionalization, via either thermal treatment or plasma treatment create many defects in the graphene, causing damage to the electronic structure and increased scattering and very difficult to control these processes. Surface-charge transfer based doping is also being investigated in various carbon materials [2-4]. It is important that the adsorption binding is not so strong that it then disrupt the graphene properties and not so weak that there is no thermal stability. However, some of these doping mechanisms also involve adhesion of organic species that are themselves large enough that they can be held firmly in place by van der Waals (or more generally nonlocal-correlation) [5] forces. Similarly, small molecules (that may by themselves desorb) may cluster, thus gaining a large physical extension that still permits it to be held in place by weak chemisorption. With weak chemisorption one will have a finite charge transfer but one will not have any significant graphene deformation [6, 7]. In effect, one achieves the analogy to modulation doping (known from semiconductor-hetero structure designs) and can thus suppress scattering.

For example, metal chlorides [8-12], organic dopants [13-17] and volatile oxidants $\left(\mathrm{HCl}, \mathrm{H}_{2} \mathrm{SO}_{4}, \mathrm{HNO}_{3}, \mathrm{SOCl}_{2}\right)$ have been studied in recent years as viable dopants for surface transfer doping of graphene [18-21]. Kasry et al. [22] 
studied the effect of p-type doping on graphene using nitric acid to reduce its sheet resistance to a value closer to conventional transparent conducting oxides. They observed a reduction in sheet resistance by a factor of 3 and yielded films consisting of eight stacked layers of graphene with a sheet resistance of $90 \mathrm{ohms} / \mathrm{sq}$. and a transmittance of $80 \%$. They attribute the reduction in sheet resistance to the formation of charge transfer complex between graphene and nitric acid described by the following reaction which was proposed by Fillaux et al. [23]. Das et al. [24] dip casted pristine graphene in $\mathrm{HNO}_{3}$ solution of varying concentrations of $\mathrm{HNO}_{3}$. The work function was increased from 4.52 to $5.31 \mathrm{eV}$ causing a shift in the Fermi level in graphene which gave it a p-type doped character with increased hole concentration. Bae et al. [25] used wet chemical doping using $\mathrm{HNO}_{3}$ on 30 -inch chemical vapor deposition (CVD) graphene made using a roll to roll process and obtained films with sheet resistance of about $125 \mathrm{ohms} / \mathrm{sq}$. and an optical transmittance of $97.4 \%$. They further improved these values to about $30 \mathrm{ohms} / \mathrm{sq}$. with $90 \%$ transparency by making a doped four layer film using layer by layer stacking.

Eda et al. [26] demonstrated that $\mathrm{SOCl}_{2}$ treatment reduced sheet resistance in graphene oxide films and used it as a transparent hole collecting electrode in organic photovoltaic devices. $\mathrm{Li}$ et al. [27] studied the effect of $\mathrm{SOCl}_{2}$ doping of CVD graphene on photovoltaic properties of the graphene/silicon Schottky junction solar cell and found that it significantly improved the conductivity of graphene and improved the conversion efficiency of the cell by threefold. Later, two articles from the same laboratory investigated several dopants for applications in graphene/silicon hetero junction solar cells [28, 29]. The first article demonstrated p-type chemical doping using chloride and nitrate ions from $\mathrm{SOCl}_{2}$ and $\mathrm{HNO}_{3}$ respectively to enhance the Schottky junction in the solar cells [28]. They found that nitrate ions were better at reducing the sheet resistance and increasing the work-function for better charge separation and transport leading to an improvement in efficiency by a factor of 1.68 . The second article explored the effect of doping via four volatile oxidants namely, $\mathrm{HCl}, \mathrm{HNO}_{3}, \mathrm{H}_{2} \mathrm{O}_{2}$ and $\mathrm{SOCl}_{2}$ on the power conversion efficiency (PCE) of graphene/silicon solar cells and this study also evaluated the thermal stability [29]. They found that all of them showed an improvement in PCE with $\mathrm{SOCl}_{2}$ showing the best out of them. PCE was found to be decreased with time and $\mathrm{SOCl}_{2}$ and $\mathrm{HCl}$ cases showed better stability than the rest.

There are contracting observation reported for $\mathrm{HNO}_{3}$ and $\mathrm{SOCl}_{2}$ doping case. Cui et al. [29] reported that $\mathrm{SOCl}_{2}$ doped Graphene/Si Schottky heterojunction solar cells shows better power conversion stability than $\mathrm{HNO}_{3}$ doped case. However, Li et al., [28] demonstrated that nitrate ions from $\mathrm{HNO}_{3}$ were found to be more effective for reducing the sheet resistance and enlarging the work function of graphene for effective charge separation and transport compared to $\mathrm{SOCl}_{2}$ case. Meanwhile, Chen et al. [30] and Xie et al. [31] demonstrated effective and non-destructive p-type doping of epitaxial graphene by depositing thin films of $\mathrm{MoO}_{3}$ on its surface. Their results show that the large difference in work function between the epitaxial graphene $(4.0-4.3 \mathrm{eV})$ and $\mathrm{MoO}_{3}(6.8 \mathrm{eV})$.

In case of $\mathrm{MoO}_{3}$ different doping mechanisms are reported. Some papers discuss charger-transfer induced due to annealing [32] and some discuss $\mathrm{MoO}_{3}$ cluster formation due to multistage doping, without annealing [33-37]. Formation of metal carbide (Mo-C) with annealing at very high temperature is also reported [38]. Wu et al. [39] conducted a similar electronic structure study of interface between $\mathrm{MoO}_{3}$ and CVD graphene and observed a hole density of about $5.44 \times 10^{12} \mathrm{~cm}^{-2}$ and a lowering of Fermi level by $0.28 \mathrm{eV}$. They report that the formation of surface negative dipoles due to electron transfer from the graphene to $\mathrm{MoO}_{3}$ causes an increase in the samples work function and the presence of gap states due to oxygen vacancies significantly reduces the hole injection barrier at the interface. Hellstrom et al. [32] demonstrated strong and stable doping of graphene and CNT films using $\mathrm{MoO}_{3}$ for transparent electrodes. They observe a reduction in sheet resistance after the interaction with the $\mathrm{MoO}_{3}$, and also observed that the sheet resistance further reduced upon annealing these systems in an inert gas atmosphere. In their experiments with graphene $\mathrm{MoO}_{3}$ bilayers, they observed a reduction of sheet resistance from $465 \mathrm{ohms} / \mathrm{sq}$. to 340 per square. which was further reduced to $260 \mathrm{ohms} / \mathrm{sq}$. $24 \mathrm{~h}$ after annealing with an overall improvement by $42 \%$. Hellstrom et al. [32] have also demonstrated doping stability, noting that the sheet reduction of the conductivity was observed upon high temperature annealing. They claimed that the annealing drives the partial oxidation of the nano carbon and partial reduction of $\mathrm{MoO}_{3}$ much further than mere deposition and refer to it as activation of $\mathrm{MoO}_{3}$ towards chemical oxidation of nanocarbon which they found to be optimal around $450{ }^{\circ} \mathrm{C}$. Quite recently, Meyer et al. [33] studied the interface structure of graphene with thermally evaporated $\mathrm{MoO}_{3}$ films and found that it gave rise to large $1.9 \mathrm{eV}$ interface dipoles and a downward bending of the $\mathrm{MoO}_{3}$ conduction band towards the Fermi level of graphene causing a nearly ideal alignment of the transport levels. The Fermi level shifted downwards by $0.25 \mathrm{eV}$ manifesting as a p-type doping with sheet resistance values lower than $50 \mathrm{ohms} / \mathrm{sq}$. for few layer graphene. Santiago et al. [40] reported a two order improvement of electrical properties carbon nano tubes after evaporation with $\mathrm{MoO}_{3}$. There is no annealing effect in these carbon nanotubes- $\mathrm{MoO}_{3}$ systems; merely changing thickness gave rise to improve the electrical conductivity. It is therefore interesting to determine if different oxidation states on $\mathrm{MoO}_{\mathrm{X}}$ are created [7] due to the formation of $\mathrm{MoO}_{\mathrm{X}}$ clusters. 
The current work seeks and tests techniques that can effectively dope the graphene while causing minimal to no damage to the graphene structure. This is necessary for its application in advanced electronics but we have found no other comparative studies of non-destructive charge transfer doping of graphene using three kinds of dopants thionyl chloride $\left(\mathrm{SOCl}_{2}\right)$, nitric acid $\left(\mathrm{HNO}_{3}\right)$ and molybdenum trioxide $\left(\mathrm{MoO}_{3}\right)$ in the literature. Our work has the following key objectives: (i) establish protocols for non-destructively doping graphene using charge transfer doping to improve its conductivity for application in electronics; and (ii) determine the most effective doping scenario for applications in advanced electronics by comparing the merits of using $\mathrm{SOCl}_{2}, \mathrm{HNO}_{3}$ and $\mathrm{MoO}_{3}$ as p-type dopants for graphene. Finally, we comment on the most effective doping scenario for applications in advanced electronics by comparing their merits.

\section{Experimental section}

\subsection{Methods: synthesis of graphene, chemical doping and theoretical studies}

The graphene was grown using a thermal CVD process with copper as the catalyst. Copper foil was used as the growth substrate which was first cleaned and then subjected to high temperatures in a thermal CVD setup in the presence of a carbon source to result in graphene growth. Three kinds of dopants $\mathrm{SOCl}_{2}, \mathrm{HNO}_{3}$ and $\mathrm{MoO}_{3}$ were used for chemical doping experiment. All reagents were received from SigmaAldrich and used as-received without further purification. More details about the thermal CVD process and chemical doping are given at Supporting Information section.

Our theoretical analysis is based on first-principle density functional theory (DFT), using the van der Waals density functional (vdW-DF) method [41-43]. We use the consistent-exchange vdW-DF-cx version [44] as implemented in the Quantum Espresso (QE) code package [45]. We determine the adsorption-induced doping by computing the charge distribution on each atom using Bader analysis [46]. We also perform Car-Parrinello molecular dynamics (CPMD) simulations [47], again using QE package, to track the stability of binding and cluster formulation.

The graphene is modelled by a $5 \times 5$ unit cell containing 50 atoms and a vacuum region of at least $12 \AA$. Ultrasoft pseudopotential with a cutoff energy of 40 Ry and a $4 \times 4 \times 4$ Monkhorst-Pack k-point sampling is used in our DFT simulations. All atoms are allowed to move till the interatomic forces are smaller than $0.008 \mathrm{eV} / \AA$.

For all of the CPMD simulations the Brillouin zone integration is performed using the Gamma-point only. The equation of motion is integrated using a timestep of 8 a.u. and an electron mass of 400 a.u. All adsorption systems are heated up to $350 \mathrm{~K}$ in canonical (NVT) ensembles with Nosé-Hoover thermostats. The stability of the adsorption systems is then studied in microcanonical (NVE) ensembles.

\subsection{Characterization}

Raman spectroscopy was carried out on the graphene samples before and after the doping process. A Raman microscope with a $638 \mathrm{~nm}$ laser along with a 1200 lines $/ \mathrm{mm}$ grating was used for all measurements. The morphology of graphene was characterized by scanning electron microscopy (SEM). This process was carried out in a Zeiss Supra 60 VP instrument. X-ray photoelectron spectroscopy (XPS) was used to measure the composition, chemical and electronic states of the graphene samples before and after the doping process. Sheet resistance measurement was carried out in a 4-point probe setup (CMT-SR2000N). Temporal and temperature stability study of the doped graphene as a function of time was evaluated by measuring the sheet resistance of the doped samples over an extended period.

\section{Results and discussion}

In present study, CVD graphene has been non-destructively doped using a surface-charge transfer method to improve its conductivity for application in electronics. The efficacy of three kinds of dopants $\mathrm{SOCl}_{2}, \mathrm{HNO}_{3}$ and $\mathrm{MoO}_{3}$ were evaluated with respect to the extent of improvement in the conductivity of graphene, effect on the graphene quality and most importantly, their stability with increasing time and temperature. The latter criteria is essential to determine the viability of their use in electronic applications. So, we comment on the most effective doping scenario for applications in advanced electronics by comparing their merits after careful evaluation.

The graphene samples were grown using thermal CVD on the copper foil, its morphology was observed using SEM. Details of the synthesis and transfer were given at the Supplementary Information section (Supplementary Figs. 1-5). Raman spectroscopy was performed on the transferred graphene samples to check the quality of the transferred graphene and indicate that the graphene obtained is predominantly single layer. Raman spectrum of one of the transferred graphene samples along with a map of ratios between the 2D and G peak intensities showed at Supplementary Fig. 6. The $\mathrm{D}$ band around $1340 \mathrm{~cm}^{-1}$ indicates the level of disorder or defects in the graphene. A perfect crystal of graphene would not have a D band. As seen from the figure, the $\mathrm{D}$ peak is quite low and shows that the graphene obtained is of good quality. The two most prominent features expected in graphene, the $\mathrm{G}$ and the $2 \mathrm{D}$ bands are also visible. The $\mathrm{G}$ 
band around $1601 \mathrm{~cm}^{-1}$ arises due to the in-plane stretching of carbon-carbon bonds and is a signature for all carbon allotropes with $s p^{2}$ bonding. The most intense feature is the 2D band around $2645 \mathrm{~cm}^{-1}$ and is the second order of the $\mathrm{D}$ peak. The ratio between the $2 \mathrm{D}$ and $\mathrm{G}$ peak intensities (I2D/IG) are above 1 in all cases and most of them are close to or above 2 . This ratio above 1 , along with the narrow FWHM of the 2D band close to $30 \mathrm{~cm}^{-1}$ indicate that the graphene obtained is predominantly a single layer and verify the observations using SEM. It is worth mentioning that although the same process was used to prepare the graphene samples, there was some degree of variation in the quality of graphene obtained and the Raman spectra between samples. Therefore, each sample was analyzed individually before and after doping.

After the graphene samples were synthesized, they were doped with three kinds of dopants $\mathrm{SOCl}_{2}, \mathrm{HNO}_{3}$ and $\mathrm{MoO}_{3}$, and analyzed to observe the effect of doping through various characterization techniques. The details of the doping were given at method section and supplementary information section. The morphology of the graphene films after the different doping treatments were observed using SEM. Figure $1 \mathrm{a}, \mathrm{b}$ and $\mathrm{c}, \mathrm{d}$ show SEM images of graphene samples after the $\mathrm{SOCl}_{2}$ and $\mathrm{HNO}_{3}$ treatment respectively. The graphene flakes and grain boundaries are visible. An organic substance was observed after the graphene was exposed to vapors. The wrinkles that were observed in the untreated graphene seem to have reduced after the vapor treatment process in both the cases. There were no major rips or tears observed in the films for either of the cases showing that the process of vapor doping is not strong enough to cause major tears to the graphene as in other chemically converted graphene. The morphology of graphene after $\mathrm{MoO}_{3}$ deposition is showed at Fig. 1e, f. The $\mathrm{MoO}_{3}$ seems to have covered all of the graphene while the grain boundaries and wrinkles of the graphene underneath are still visible. It also appears that the $\mathrm{MoO}_{3}$ deposition is slightly more aggregated near the wrinkles and is more visible. SEM images of the sample after annealing have been shown in Fig. $1 \mathrm{~g}-\mathrm{i}$. At a lower magnification, the graphene does not look much different compared to the undoped graphene. However, on closer examination the $\mathrm{MoO}_{3}$ has formed smaller islands which uniformly cover the underlying graphene. This was not observed in the samples after deposition and indicates that the $\mathrm{MoO}_{3}$ undergoes dewetting from the graphene film during the annealing.

Sheet resistance for the samples was measured for three samples before and after doping for each dopant species. The sheet resistance values before and after $\mathrm{SOCl}_{2}$ treatment in three different samples that have been numbered for convenience (Fig. 2a). It can be seen that a reduction in sheet resistance is consistently observed in all three samples. Sample 1.1 shows the biggest reduction of $41 \%$ while the smallest reduction is $37 \%$ in sample 1.3 . The average reduction in sheet resistance from these three samples is about $38 \%$. Figure $2 \mathrm{~b}$ highlights the effects found in the case of $\mathrm{HNO}_{3}$ treatment. Here there is a significant reduction in sheet resistance consistently observed with the biggest reduction of $73 \%$ and an average reduction of about $70 \%$ which is significantly more than the $\mathrm{SOCl}_{2}$ case. Figure $2 \mathrm{c}$ shows the comparison between sheet resistance values for three samples before $\mathrm{MoO}_{3}$ deposition, after deposition and after annealing. It can be seen that for all three samples, the sheet resistance values initially increase after deposition. This is probably because the graphene materials are not coated with $\mathrm{MoO}_{3}$ immediately after they are transferred so they could be slightly degraded or contaminated when exposed in air. This is verified by experiment results that shows the graphene resistance change with different storage duration, as shown in Fig. S11 in the Supporting Information. However, the sheet resistance values reduce significantly after annealing at $450{ }^{\circ} \mathrm{C}$ in argon atmosphere.

The previous reports on graphene doping with $\mathrm{MoO}_{3}$ all report a reduction in sheet resistance soon after the deposition, while Hellstrom et al. [32] reported a more significant reduction after annealing. A reduction in sheet resistance was not observed immediately in any of the samples and was only observed after the annealing process. The annealing process possibly helped improve the interaction between the graphene and the $\mathrm{MoO}_{3}$ causing the charge transfer and thus the reduction in sheet resistance. The biggest reduction in sheet resistance was $59 \%$ while the remaining two samples showed a reduction in sheet resistance by about $49 \%$ with an average reduction of about $52 \%$. This variation in the extent of reduction in sheet resistance between the samples despite the same doping process could be due to the variation in the initial quality of the graphene and possibly some minor changes during the processing.

Raman spectroscopy was carried out on the graphene samples before and after the doping process to see the effect of doping on the graphene through changes in the spectra. Raman data obtained from the samples with the highest improvements (for each of the three doping candidates) are shown in the figures in this section. Figure $3 a$, $b$ shows the Raman spectra of the samples before and after the $\mathrm{SOCl}_{2}$ where the Fig. $3 b$ shows the $G$ band of the same spectra. One of the prominent features of p-type doping in graphene is an upshift of the $\mathrm{G}$ band. This can be clearly observed in this sample where the peak position shifts from $1599 \mathrm{~cm}^{-1}$ before doping to $1606 \mathrm{~cm}^{-1}$ showing an upshift by $7 \mathrm{~cm}^{-1}$. This shift in the $\mathrm{G}$ band is an indication of p-type doping and is known to depend on the amount of charge removed per carbon atom. We note that greater shift implies more charge is withdrawn, although this dependence is not linear. This prominent shift in G peak was observed in all the samples. A reduction in I2D /IG ratio was observed along with 

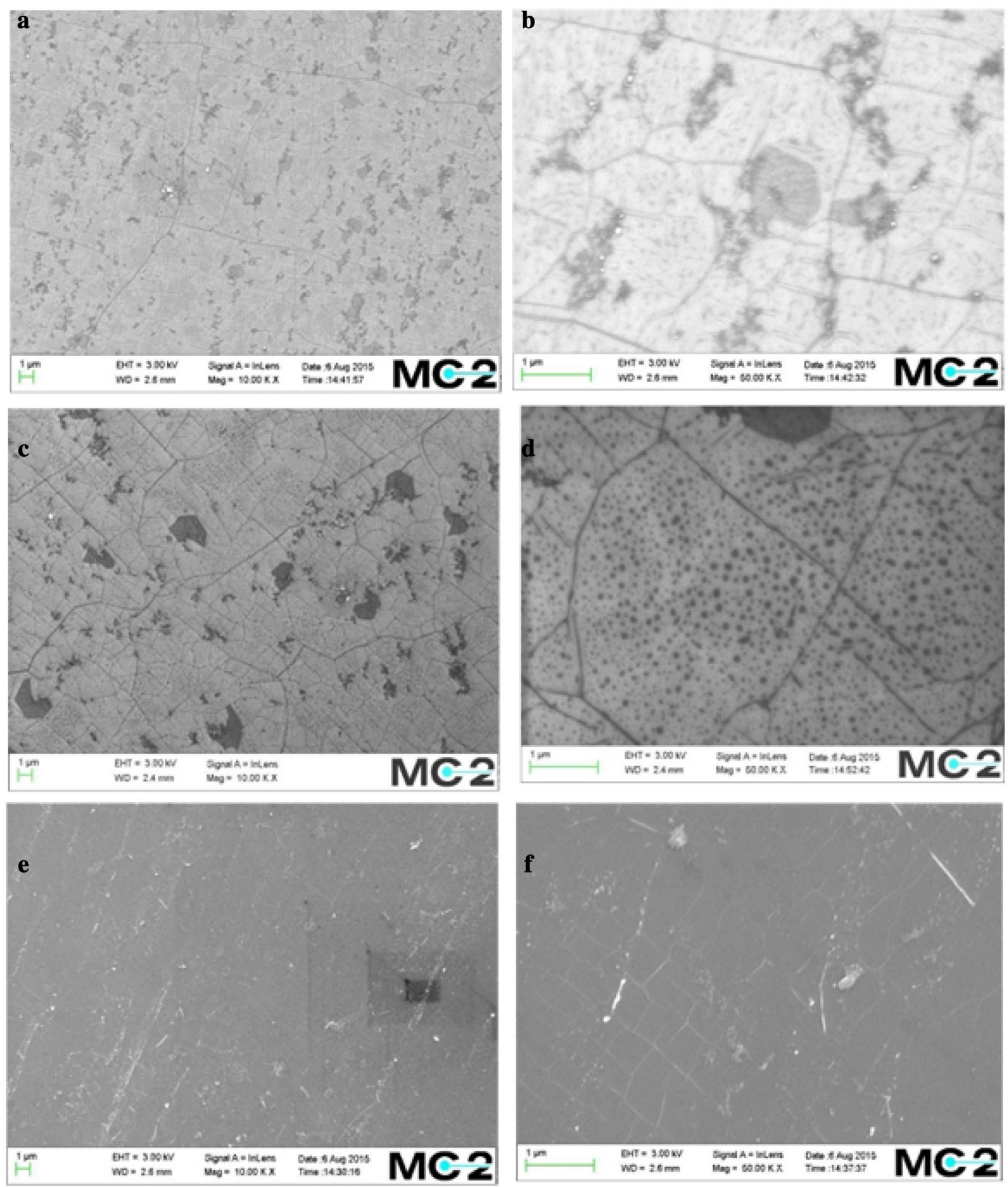

Fig. 1 SEM images of graphene treated with three different dopants with different magnification; $\mathrm{SOCl}_{2}$ vapors (a $10 \mathrm{kx}$ and b $50 \mathrm{kx}$ );

tion (e $10 \mathrm{kx}$ and $\mathbf{f} 50 \mathrm{kx}$ ); after $\mathrm{MoO}_{3}$ deposition and annealing (g $10 \mathrm{kx} \mathrm{h} 50 \mathrm{kx}$ i $150 \mathrm{kx}$ ) 

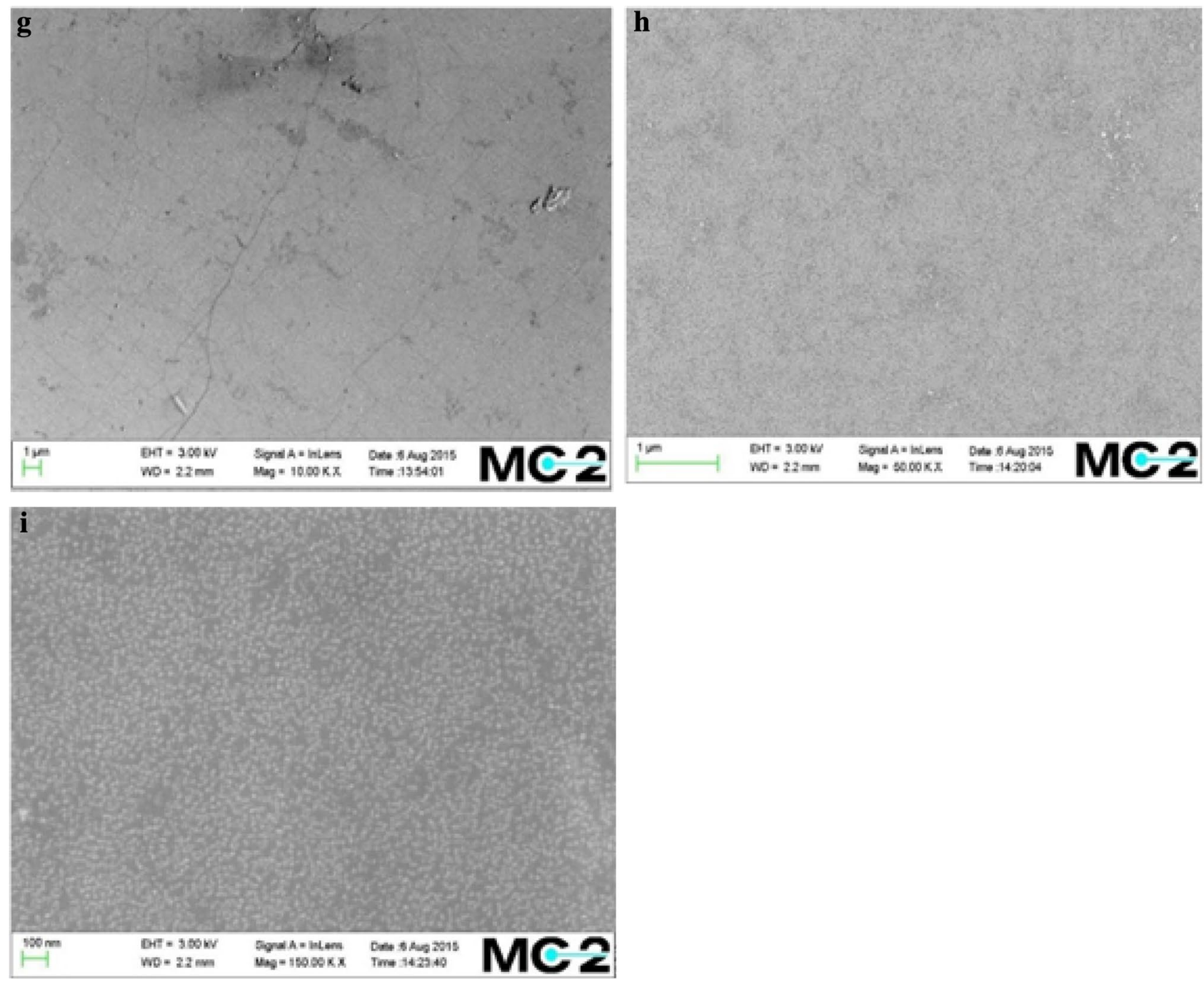

Fig. 1 (continued)
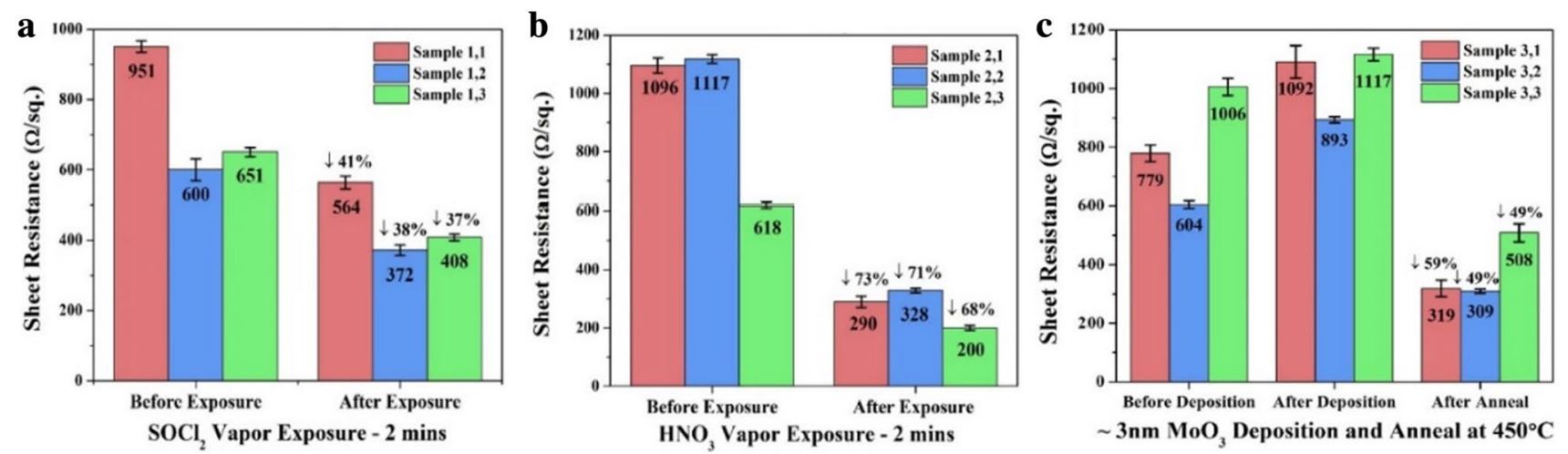

Fig. 2 Figure showing the change in sheet resistance of graphene sample after doping. a $\mathrm{SOCl}_{2}, \mathbf{b} \mathrm{HNO}_{3}$, and $\mathbf{c} \mathrm{MoO}_{3}$. In case of $\mathrm{SOCl}_{2}$ and $\mathrm{HNO}_{3}$ sheet resistance values reduce after exposure, how- ever in case of $\mathrm{MoO}_{3}$, the sheet resistance values are increased after deposition and reduced significantly after annealing at $450{ }^{\circ} \mathrm{C}$ in argon atmosphere. (Color figure online) 
Fig. 3 Raman spectra of graphene sample before and after $\mathrm{SOCl}_{2}$ treatment: a full spectra, b $\mathrm{G}$ band region. (Color figure online)

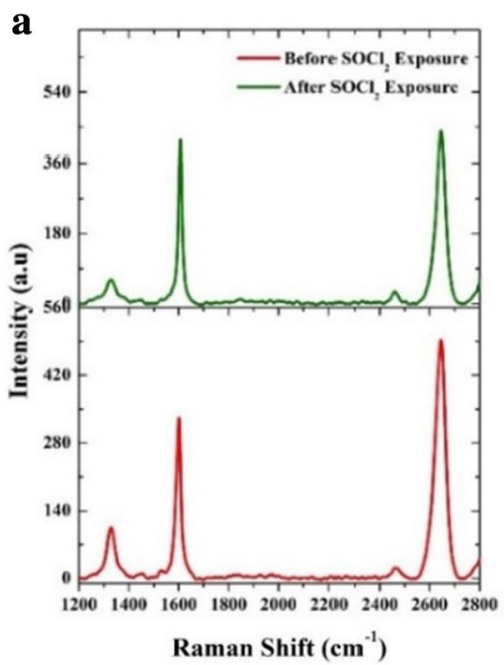

a narrowing of the $\mathrm{G}$ band as can be seen from the Raman spectra which is expected from doping graphene due to suppression of the $2 \mathrm{D}$ band. Interestingly, it was observed that the ID/IG ratio reduced from 0.32 to 0.14 after the $\mathrm{SOCl}_{2}$ treatment which corresponds to fewer defects in the graphene. This reduction was also observed to varying degrees in the other samples treated with $\mathrm{SOCl}_{2}$ which suggests that the treatment reduces defects in the graphene and confirms its non-destructive nature.

Figure 4 compares the Raman spectra of the sample before and after $\mathrm{HNO}_{3}$ treatment. In this case, the upshift in the $\mathrm{G}$ band position is even more pronounced where it shifts from 1601 to $1610 \mathrm{~cm}^{-1}$ with an increase of about $9 \mathrm{~cm}^{-1}$. A similar upshift was observed in the other samples treated with $\mathrm{HNO}_{3}$ and was always larger than that observed in the $\mathrm{SOCl}_{2}$ treated samples. Therefore more significant charge transfer effect takes place in this case and agrees with the extent of reduction in sheet resistance when comparing both the vapor doping scenarios. There b

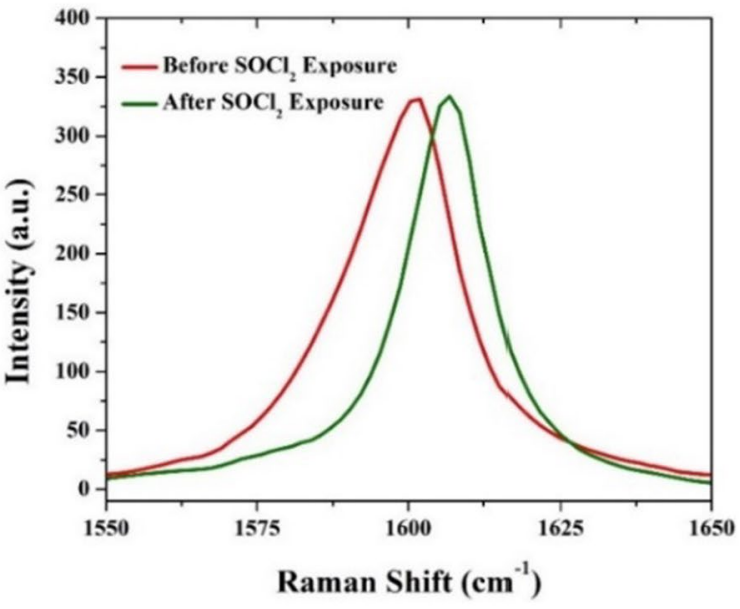

was no major changes in the ID/IG ratio which suggests that the $\mathrm{HNO}_{3}$ treatment does not introduce significant defects in the graphene. Figure 5 shows the Raman spectra of a graphene sample before $\mathrm{MoO}_{3}$ deposition, after deposition and after annealing. It can be seen that although there is a minor upshift of the $\mathrm{G}$ band after the deposition from 1604 to $1605 \mathrm{~cm}^{-1}$, the major shift by $15-1619 \mathrm{~cm}^{-1}$ was observed after annealing indicating that the charge transfer takes place during annealing and agrees with the results of the sheet resistance measurements. Another interesting feature is the broadening of the $G$ band and a second component seems to exist in the $G$ band after annealing. The origin of this second component is unclear and is associated with either deposition or adsorption of some material on the graphene, strain in graphene or presence of local charge inhomogeneity. A reduction of the I2D/IG ratio was also observed which is expected. There seems to be an increase in the ID/IG which suggests a slight increase in the defects in the graphene. However,
Fig. 4 Raman spectra of graphene sample before and after $\mathrm{HNO}_{3}$ treatment: a full spectra, b $\mathrm{G}$ band region. (Color figure online)

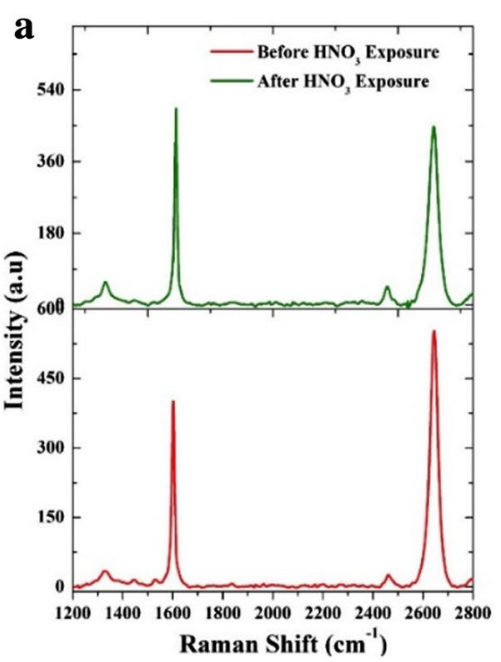


Fig. 5 Raman spectra of graphene sample before and after $\mathrm{MoO}_{3}$ deposition and annealing: a full spectra, $\mathbf{b} \mathrm{G}$ band region. (Color figure online)

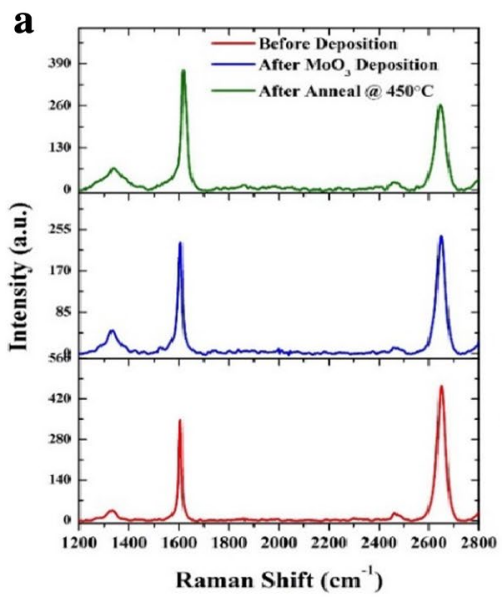

b

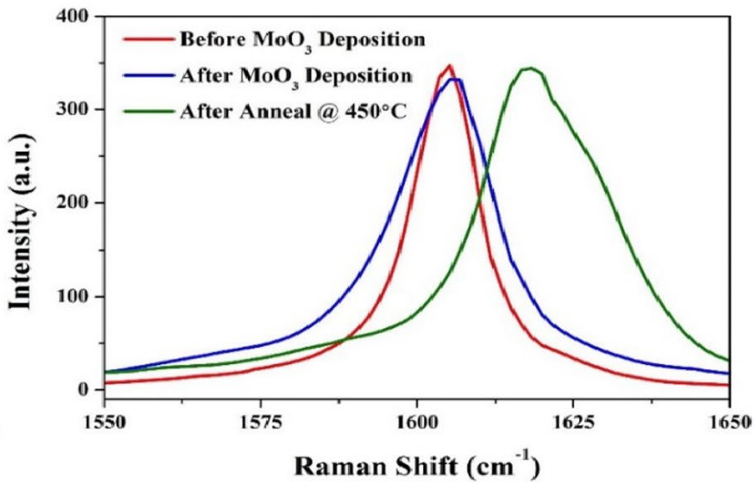

this was not observed in all the samples and would require further investigation.

$\mathrm{X}$-ray photoelectron spectroscopy also used to confirm the presence of the dopant species and to observe their interaction. The XPS also allowed us to track shifts in the Fermi level of graphene. We studied XPS obtained from four different samples; undoped graphene, $\mathrm{SOCl}_{2}$ treated graphene, $\mathrm{HNO}_{3}$ treated graphene, $\mathrm{MoO}_{3}$ coated and annealed graphene. Precise quantification of the XPS spectra allows us to obtain composition but this approach also requires very exact fitting of the peaks with proper constraints. Survey spectra obtained from each of our four samples are given at Fig. 6. The predominant peaks have been labeled and the respective elements that would be expected were observed in each of the cases. Carbon, oxygen, and silicon peaks were observed in all the samples from the graphene and the underlying $\mathrm{SiO}_{2}$ substrate. Moreover, The $\mathrm{Cl} 1 s$ and $\mathrm{Cl} 2 p$ peaks were visible in the sample treated with $\mathrm{SOCl}_{2}$ vapors while $\mathrm{N} 1 s$ peak was detected in the sample treated with $\mathrm{HNO}_{3}$ vapors. The sample doped with $\mathrm{MoO}_{3}$ shows the Mo3s, Mo3 $p$ and Mo3d peaks. Apart from the expected peaks, there were also strong unexpected peaks such as the fluorine and aluminum peaks which appear on all the samples the origin of which is unclear. A high resolution $\mathrm{C} 1 s$ spectra were acquired for each of the sample.

The doped samples show varying degrees of shift when compared to the pristine sample (Fig. 7). The $\mathrm{C} 1 s$ binding energy for the undoped sample is about $284.69 \mathrm{eV}$, while that of the $\mathrm{SOCl}_{2}$ has downshifted by $0.40-284.29 \mathrm{eV}$. In the case of the sample doped with $\mathrm{MoO}_{3}$, the binding energy of $\mathrm{C} 1 s$ is centered around $284.40 \mathrm{eV}$ showing a shift of $0.29 \mathrm{eV}$. However, a very small shift of $0.02 \mathrm{eV}$ for $\mathrm{HNO}_{3}$ doped sample was observed which is much smaller than expected. Therefore, the XPS measurements would need to be repeated and fitted carefully to extract precise changes in Fermi level of the graphene after doping. A high resolution spectra of $\mathrm{Cl} 2 p, \mathrm{~N} 1 s$ and Mo3d levels acquired from the samples doped with $\mathrm{SOCl}_{2}, \mathrm{HNO}_{3}$ and $\mathrm{MoO}_{3}$ respectively (Fig. 8). The $\mathrm{Cl} 2 p$ peak around $199 \mathrm{eV}$ seems to consist two peaks, one near $198.5 \mathrm{eV}$ which corresponds to the presence of $\mathrm{Cl}^{-1}$ ions while the other around $200.3 \mathrm{eV}$ which corresponds to $\mathrm{Cl}-\mathrm{C}$ covalent bonds. The $\mathrm{N} 1 s$ spectra from the $\mathrm{HNO}_{3}$ doped sample shows two main peaks, a large peak at $407.2 \mathrm{eV}$ corresponding to $\mathrm{C}-\mathrm{N}$ covalent bonding and another one around $400 \mathrm{eV}$ which corresponds to the $\mathrm{NO}_{3}{ }^{-1}$ ionic bonds. These results suggest that the $\mathrm{C}-\mathrm{Cl}$ and $\mathrm{C}-\mathrm{N}$ interactions take place due to the doping process and contribute to the enhancement in the electronic transport [24, $28,48]$. The Mo- $3 d$ peak seems to consist of three main peaks. The peaks around 232.6 and $235.8 \mathrm{eV}$ correspond mainly to $\mathrm{Mo}^{6+}$ oxidation states and possibly contain smaller components from $\mathrm{Mo}^{5+}$. The peak around $229.8 \mathrm{eV}$ corresponds to the $\mathrm{Mo}^{4+}$ oxidation states. Previous works on doping of graphene with $\mathrm{MoO}_{3}$ where the samples were not subjected to annealing did not show any peak corresponding to $\mathrm{Mo}^{4+}$ oxidation states $[30,32,33]$. These $\mathrm{Mo}^{4+}$ oxidation states seem to become visible only after the annealing which strongly suggests reduction of $\mathrm{MoO}_{3}$, indicating that it receives electrons from the graphene, thus causing more efficient charge transfer. These results agree with the observation by Hellstrom et al. [32]. Moreover, no major evidence of Mo-C bonding was observed from the Mo3d spectra.

The temporal and temperature stability of the doped graphene as a function of time was evaluated by measuring the sheet resistance of the doped samples over an extended period. The results for the doping stability over time are reported at Fig. 9. We find that samples treated with $\mathrm{SOCl}_{2}$ show the least stability with time. In fact it quickly reaches $99.6 \%$ of the initial sheet resistance value before doping. $\mathrm{HNO}_{3}$ also shows a poor short term stability: while the initial reduction in sheet resistance is large, it goes up to $56.8 \%$ of the undoped value in just 2 days. However, thereafter it remains fairly stable beyond that reaching $66.3 \%$ of the undoped value at the end of 4 weeks. The increase in 

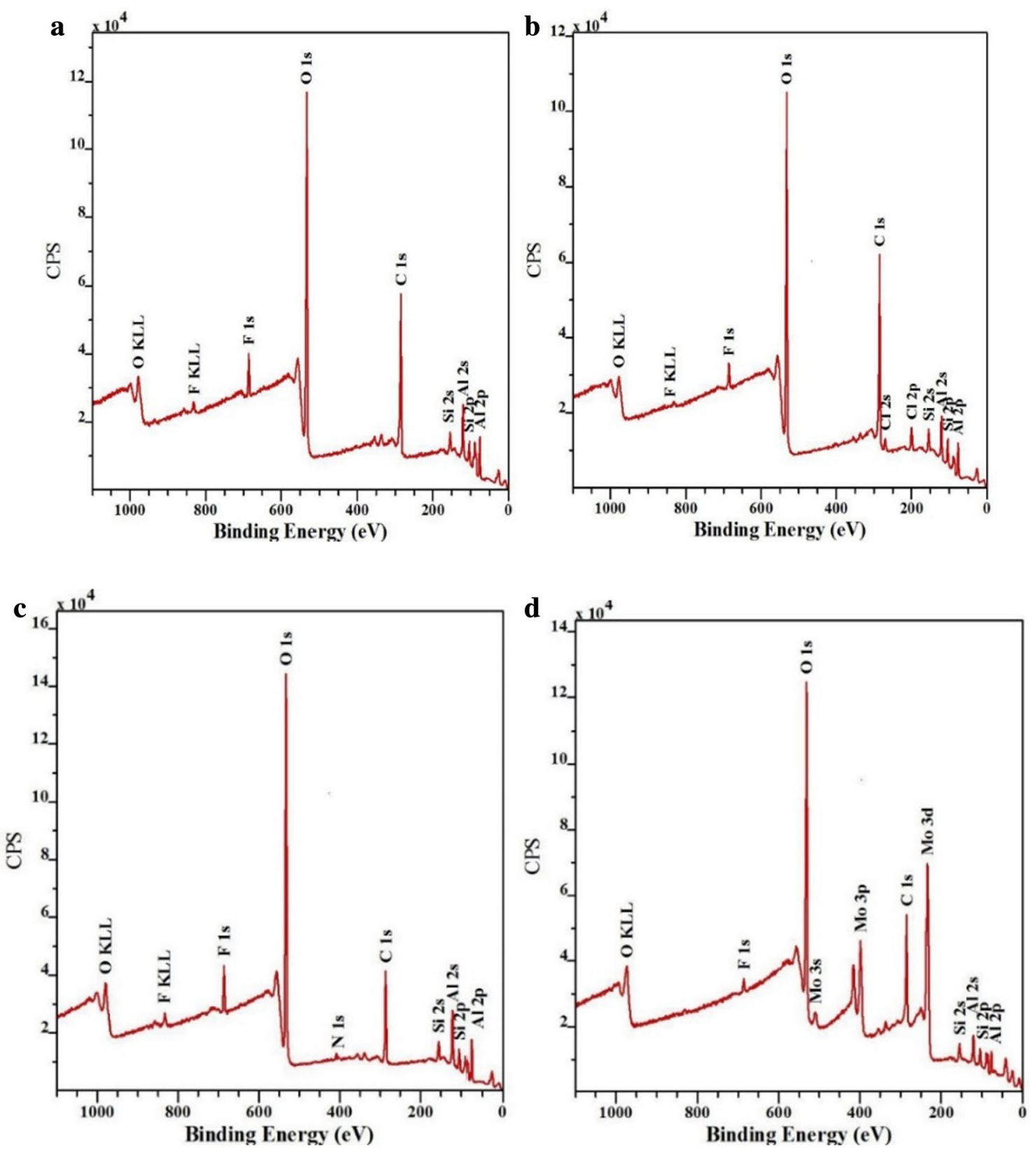

Fig. 6 The XPS survey spectra of pristine graphene and after doping. a Pristine, $\mathbf{b} \mathrm{SOCl}_{2}, \mathbf{c} \mathrm{HNO}_{3}$ and $\mathbf{d ~ M o O} \mathrm{M}_{3}$

sheet resistance with time is possibly due to reaction with the moisture in the air and also desorption of the dopants from the graphene to some extent. This could be reduced by adding a protective layer on top of the graphene after doping, however it would lead to other complications during patterning and depositing contacts for electronic applications.

The sample doped with $\mathrm{MoO}_{3}$ shows fairly good stability with time where the sheet resistance increases gradually to $63 \%$ of the undoped value at the end of 4 weeks and $66 \%$ at the end of 6 weeks. In this case, the increase in sheet resistance is predominantly due to the effect of moisture in the atmosphere. It is worth mentioning that some of the increase in sheet resistance may be due to the damage caused by the probes during each measurement which is why the measurement was not carried out very often. This possible damage due to the probes could be avoided by depositing metal contacts on the graphene instead of using the probes directly on the graphene.

Figure 10 shows a quantification of doping stability with temperature. We track the percentage increase in sheet resistance for different dopants with increasing temperatures. The figure reports the percentage increase in sheet resistance which is plotted as a function of temperature. The percentage increase in sheet resistance is 


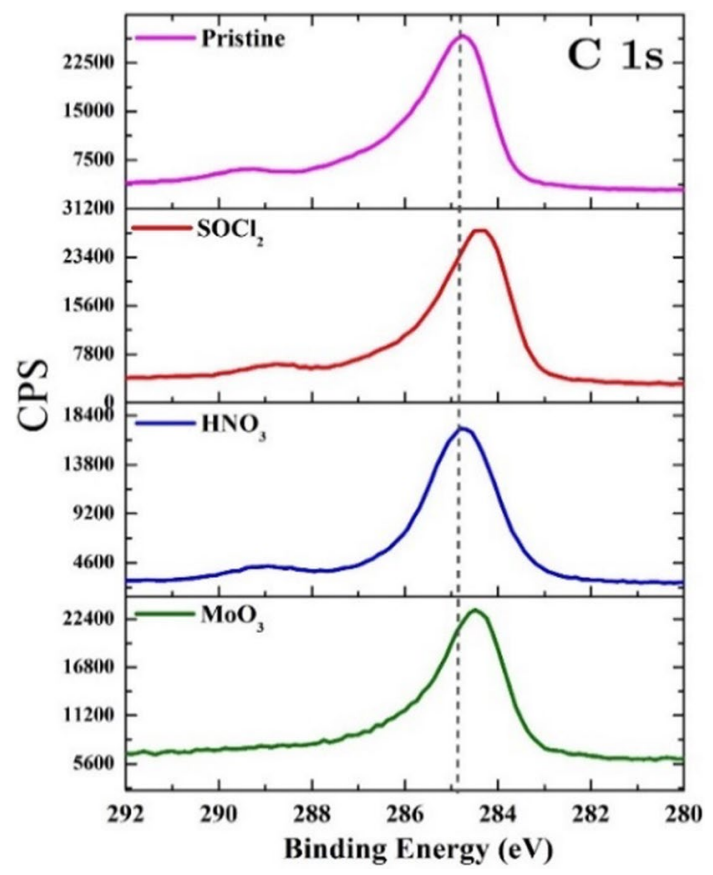

Fig. 7 High resolution XPS spectra of the four samples stacked for comparison. Top to bottom: Pristine, $\mathrm{SOCl}_{2}, \mathrm{HNO}_{3}$ and $\mathrm{MoO}_{3}$

much more pronounced for samples doped with $\mathrm{HNO}_{3}$ and $\mathrm{SOCl}_{2}$ than with $\mathrm{MoO}_{3}$. The $\mathrm{SOCl}_{2}$ doped sample reached $43.2 \%$ of its initial doped value while $\mathrm{HNO}_{3}$ doped sample reached around $40 \%$ at $200{ }^{\circ} \mathrm{C}$. This increase in sheet resistance is attributed to desorption of the dopants from the graphene at elevated temperatures. It should be noted that although the percentage increase in both these cases is quite similar, the initial reduction in the case of $\mathrm{HNO}_{3}$ is significantly larger and therefore the increase in the value of sheet resistance when compared to the undoped value is less significant than for the $\mathrm{SOCl}_{2}$ doped sample. The effect of temperature on the sheet resistance is not very significant for the samples doped with $\mathrm{MoO}_{3}$ where it reaches only $12.4 \%$ of the initial doped value. This shows that the samples doped with $\mathrm{MoO}_{3}$ has much better stability when compared to the other two doping scenarios.

\subsection{Theoretical investigation}

We also theoretically investigated the mechanisms of graphene doping by $\mathrm{HNO}_{3}, \mathrm{SOCl}_{2}$ and $\mathrm{MoO}_{3}$ molecules. Our focus was on understanding the charge transfer effects that follow the weak chemisorption of these molecules on graphene. First, we simulated the adsorption structures in DFT and tracked the stability using CPMD performed at $350 \mathrm{~K}$.

Figure 11 reports the results of our CPMD simulations for $\mathrm{HNO}_{3}$ on graphene. We find that there will be a dissociation of hydrogen or of hydrogen and oxygen atoms leaving $\mathrm{NO}_{3}$ or $\mathrm{NO}_{2}$ molecules to diffuse on the graphene. Below, we also document that the $\mathrm{NO}_{\mathrm{x}}$ adsorption causes charge transfer, i.e., that the residual molecules are technically chemisorbed. However, the diffusion documented in the CPMD studies reflect a weak chemisorption nature for the $\mathrm{NO}_{\mathrm{x}}$-graphene system and we do, in fact, find limited adsorption energies (per molecule or molecular unit) in all of the cases. The adsorption energy is calculated from $E_{a d s}=-\left(E^{g r+m o l}-E^{g r}-E^{m o l}\right)$, where $E^{g r+m o l}$ is the adsorption system energy, $E^{g r}$ and $E^{m o l}$ are the energy of isolated graphene and molecule. Regular chemical-bond formation does not account for behavior in the set of investigated adsorption cases and use of the vdW-DF method (instead of traditional semilocal DFT) is important for our theoretical characterizations.

Figure 12 summarizes our study of weak chemisorption of $\mathrm{MoO}_{3}$ on graphene. In this study, we simulated the adsorption of $\mathrm{a} \mathrm{MoO}_{3}$ single molecule, a $\mathrm{MoO}_{3}$ dimer as well as of three- and four-molecule clusters. Our CPMD simulations indicates that all $\left(\mathrm{MoO}_{3}\right)_{\mathrm{n}}$ adsorption structures are stable at $350 \mathrm{~K}$. Our CPMD results from the four $\mathrm{MoO}_{3}$ weak chemisorption problems further suggest that are significant clustering energies to drive formation of large adsorbate clusters and (at sufficient deposition) even assembly of $\mathrm{MoO}_{3}$ coatings. Having identified optimal
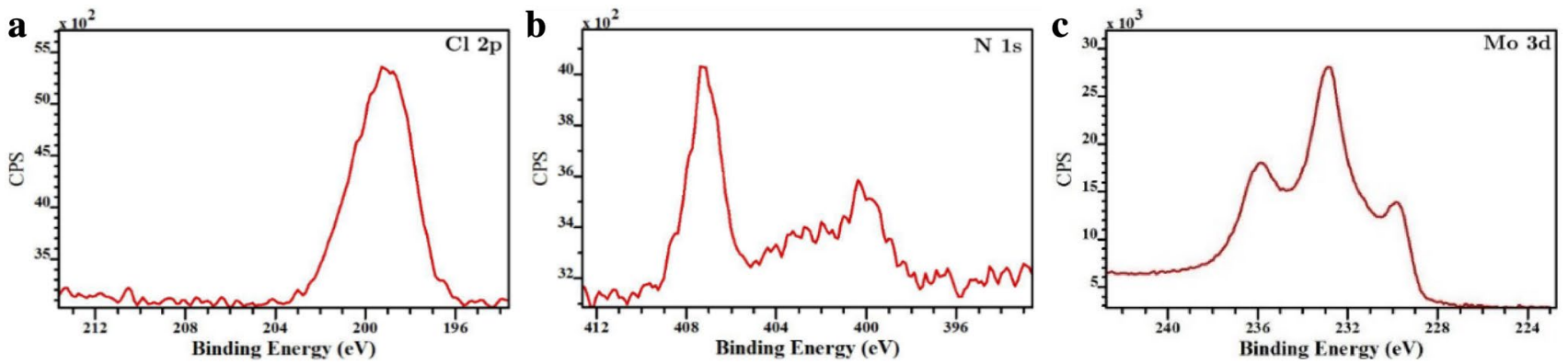

Fig. 8 High resolution XPS core level spectra for $\mathrm{Cl} 2 p, \mathrm{~N} 1 s$ and $\mathrm{Mo} 3 d$ obtained from samples doped with the respective dopant species. a Cl2 $p$ $\mathrm{SOCl}_{2}$ treated, $\mathbf{b} \mathrm{N} 1 s-\mathrm{HNO}_{3}$ treated and $\mathbf{c} \mathrm{Mo} 3 d-\mathrm{MoO}_{3}$ deposition and annealing 


\section{Temporal Stability}

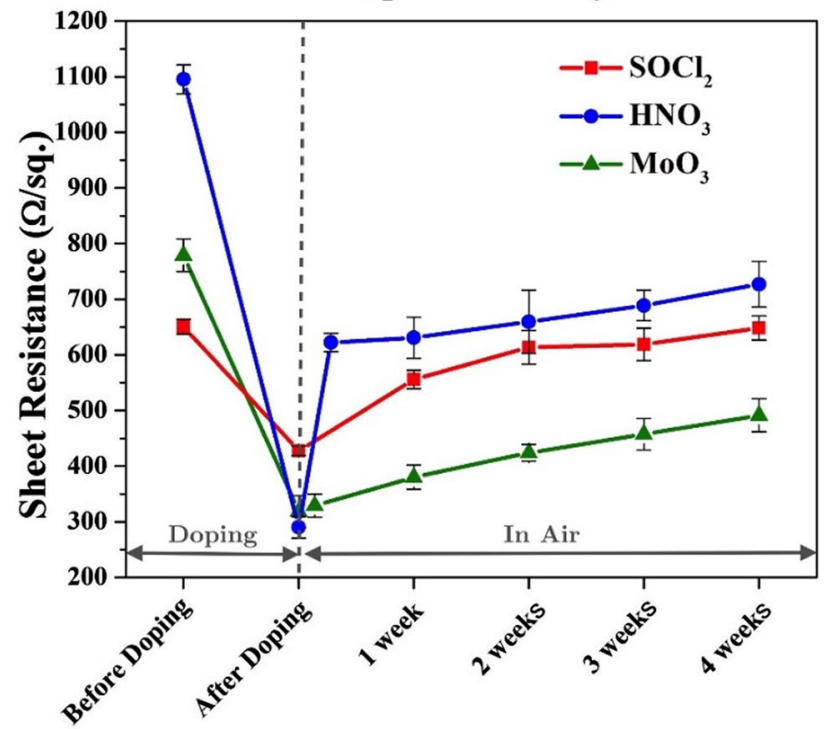

Fig. 9 Variation of sheet resistance with increasing time of the doped graphene samples

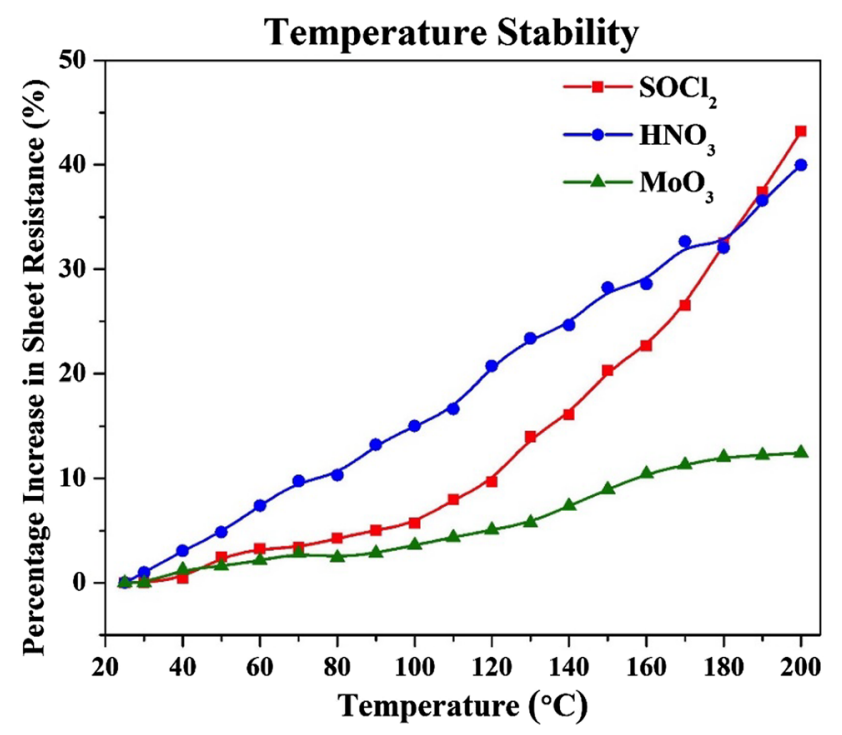

Fig. 10 Variation of sheet resistance with increasing temperature of the doped graphene samples

adsorption configurations, we extract total energies in the four systems and, in turn, define energy differences that characterize clustering among weakly chemisorbed $\mathrm{MoO}_{3}$ on graphene. This clustering cohesive energy is calculated from $E_{c c}=-\left(E_{a d s}^{g r+\left(\mathrm{MoO}_{3}\right)_{n}}-n E_{a d s}^{g r+\mathrm{MoO}_{3}}\right) / n$ and grows with the cluster size: it is $1.35 \mathrm{eV}$ per molecule for a $\mathrm{MoO}_{3}$ dimer, while it is 1.60 and $1.65 \mathrm{eV}$ per molecule for $\left(\mathrm{MoO}_{3}\right)_{3}$ and $\left(\mathrm{MoO}_{3}\right)_{4}$ clusters.
The tendency for nucleation and assembly also explains why the thermal stability of the $\mathrm{MoO}_{3}$ increases with thermal annealing: the $\mathrm{MoO}_{3}$ clustering stabilizes the adhesion. Comparing the total energy of the adsorbed systems, of the clean graphene surfaces and of the molecule in the gas phase, we define adsorption energies $E_{a d s}$ For larger adsorbates, weak chemisorption can easily provide binding in excess at the eV scale and beyond [5-7, 49]. Table 1 includes a comparison of the adhesion energy per molecules for the set of investigated dopants and dopant-cluster configurations. The table confirms that the $\mathrm{MoO}_{3}$ cluster adhesion energy exceeds $1 \mathrm{eV}$ already when reaching a three-molecular size.

Finally, our calculations permit us to track the doping and graphene modification that occurs at optimal adsorption configurations. Table 1 reports our vdW-DF-cx results for the adsorption distances, for the associated mean absolute displacement of the carbon atoms sitting immediately below the adsorbates, and for the resulting charge transfer (as asserted in a Bader analysis of the electron distribution in the optimal configurations).

Our calculations show that all three molecules can (p-) dope via weak chemisorption but also confirms that it is imperative to check stability. The $\mathrm{HNO}_{3}$ adsorption causes dissociation and we have instead analyzed this strategy as a case of $\mathrm{NO}_{2}$ and $\mathrm{NO}_{3}$ weak chemisorption. Also, given the tendency for cluster nucleation, it is more important to characterize the doping impact of $\mathrm{MoO}_{3}$ clusters instead of individual $\mathrm{MoO}_{3}$ molecules.

Table 1 shows that $\mathrm{p}$-doping efficiencies (as obtained in DFT) are in the order, $\mathrm{SOCl}_{2}<\left(\mathrm{MoO}_{3}\right)_{\mathrm{n}}<\mathrm{NO}_{2} / \mathrm{NO}_{3}$. The adsorption of $\mathrm{SOCl}_{2}$ molecules does not seem to lead to any significant charge transfer. Given the anchoring of dissociated $\mathrm{NO}_{3}$ and $\mathrm{NO}_{2}$, one can expect that the addition of $\mathrm{HNO}_{3}$ molecules produce the highest doping efficiencies. In addition, one should note that adding $\mathrm{HNO}_{3}$ molecules also causes a strong chemisorption of excess hydrogen and oxygen atoms, shown in Fig. 11c, d, that further affects the scattering (and doping).

Interestingly, our calculations show that the assembly of $\mathrm{MoO}_{3}$ clusters causes the doping nature to change: we predict a n-type doping for the (unstable) case of singlemolecule adsorption while clustering converts the $\mathrm{MoO}_{3}$ adsorption into p-doping agents. Given our documentation of a clustering driving force, our theoretical studies corroborate the finding that annealing plays a critical role in the $\mathrm{MoO}_{3}$-induced (p-)doping.

In fact, our set of theory results seem fully consistent with the experimentally observed trends in conductivities, but we can only provide a qualitative discussion subject to an assumption of the original nature of conduction in the CVD-grown samples. In general, chemical doping increases the density of free (electron or hole) charge carriers by charge transfer mechanisms that modify the Fermi 
Fig. 11 Results of molecular dynamics simulation of $\mathrm{HNO}_{3}$ adsorbed and partly dissociated on graphene. Panel (a) shows the computed temporal evolution of the distances of the adsorbate atoms to the graphene layer. The $\mathrm{HNO}_{3}$ molecules (b) dissociates to $\mathrm{NO}_{3}(\mathbf{c})$ and then $\mathrm{NO}_{2}$ (d) while the hydrogen and oxygen atoms disperse on the graphene. The various adsorbates states are indicated in panel (a). (Color figure online)
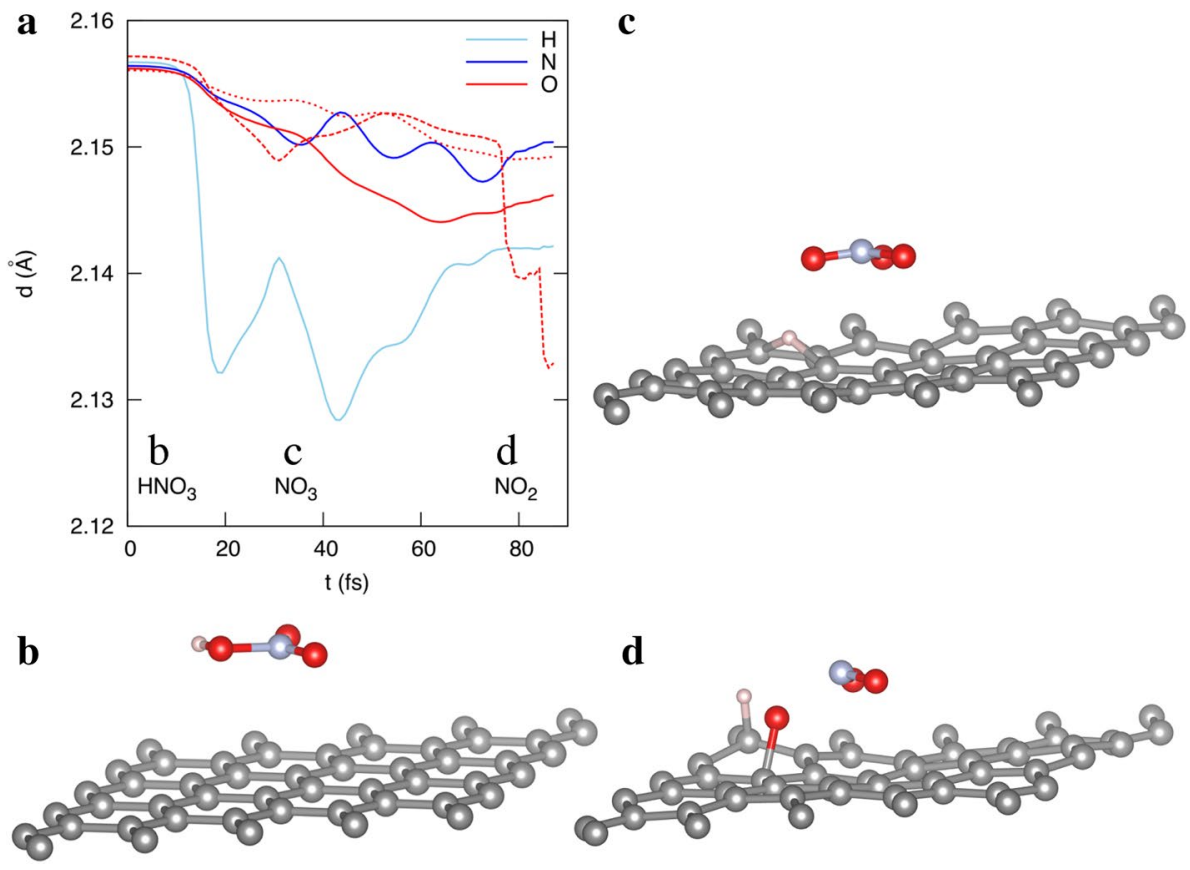

$\mathbf{a}$

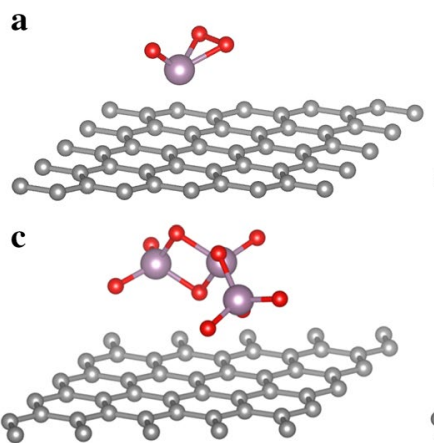

b

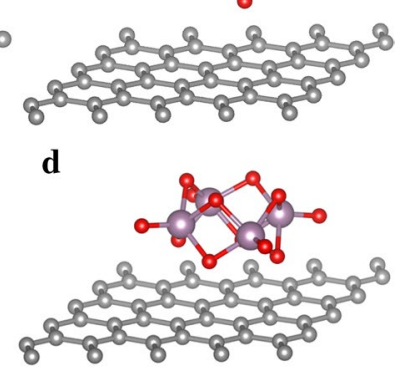

Fig. 12 Fully relaxed geometries in vdW-DF-cx of $\mathrm{MoO}_{3}$ single molecule and the first few clusters on graphene

level of the doped. However, we do not know if the CVDgrown graphene samples were $\mathrm{p}$ or $\mathrm{n}$ doped from the outset and so we cannot trace the net impact that follows with predictions of the size of p-type doping. However, we can say this: if the samples was originally p-doped, the set of theory characterizations are consistent with the measured conductivity changes.

Subject to the stated assumption, the theoretical study does confirm the experimental observation that $\mathrm{HNO}_{3}$ treatment gave rise to the highest resistance reduction but suffered from less stability. Overall, the theoretical characterization suggests that, with annealing and cluster assembly, $\mathrm{MoO}_{3}$ holds advantages in terms of both thermal stability and by having a limited deformation at a fair charge transfer. However, the weak chemisorption of adsorption of $\mathrm{MoO}_{3}$ clusters does cause some deformation

Table 1 Adsorption stabilities, configurations and doping efficiencies from first-principles simulations

\begin{tabular}{|c|c|c|c|c|}
\hline Material system & $\begin{array}{l}\mathrm{E}_{\mathrm{ads}}(\mathrm{eV} / \\
\text { molecule })\end{array}$ & $\mathrm{d}(\AA)$ & $\delta z_{C}(\AA)$ & $\begin{array}{l}\text { Doping (e } \\
\text { molecule) }\end{array}$ \\
\hline $\mathrm{SOCl}_{2}$-graphene & 0.43 & 3.07 & 0.025 & $+0.02 *$ \\
\hline $\mathrm{HNO}_{3}$-graphene & 0.29 & 3.22 & 0.020 & +0.01 \\
\hline $\mathrm{MoO}_{3}$-graphene & 1.40 & 2.46 & 0.031 & -0.21 \\
\hline $\mathrm{NO}_{3}$-graphene & & 2.96 & 0.038 & +0.51 \\
\hline $\mathrm{NO}_{2}$-graphene & & 3.42 & 0.013 & $+0.20 *$ \\
\hline$\left(\mathrm{MoO}_{3}\right)_{2}$-graphene & 0.35 & 2.87 & 0.027 & $+0.14 *$ \\
\hline$\left(\mathrm{MoO}_{3}\right)_{3}$-graphene & 0.36 & 2.82 & 0.026 & $+0.14 *$ \\
\hline$\left(\mathrm{MoO}_{3}\right)_{4}$-graphene & 0.30 & 2.79 & 0.040 & $+0.12 *$ \\
\hline
\end{tabular}

$E_{a d s}$ is the adsorption energy of the molecules on graphene. $d$ is the vertical distance from the graphene plane to the closest atom. $\delta z_{C}$ is the mean absolute displacement of the carbon atoms immediately under the adsorbates (measured from the graphene plane). The plus sign of doping corresponds to p-type doping while the minus sign corresponds to n-type doping. Stable adsorption configuration of each molecule is marked with asterisk

of the underlying graphene sheet, and it will induce electron scattering.

\section{Conclusions}

In summary, we demonstrate the efficacy of efficient, nondestructive doping of CVD graphene using thionyl chloride $\left(\mathrm{SOCl}_{2}\right)$, nitric acid $\left(\mathrm{HNO}_{3}\right)$ and molybdenum trioxide $\left(\mathrm{MoO}_{3}\right)$. The samples were characterized using microscopy, spectroscopy and electrical measurement techniques. The 
samples doped with $\mathrm{HNO}_{3}$ showed the largest (immediate) reduction in sheet resistance with an average reduction by about $70 \%$ and $\mathrm{SOCl}_{2}$ treated samples on the other hand showed the smallest reduction in sheet resistance with an average reduction by $38 \%$. Whereas $\mathrm{MoO}_{3}$ deposition on graphene increased the sheet resistance initially, this was significantly reduced after the annealing process yielding an average reduction by about $52 \%$ compared to the undoped samples. Analysis of morphology using SEM showed no severe damage to the graphene from any of the doping processes. Raman spectra on the doped samples showed an upshift in the $\mathrm{G}$ band for all the cases confirming the transfer of electrons from the graphene to the dopants, increasing the density of holes at the interface, thus effectively giving the graphene a p-type doped character.

The presence of the dopant species on the graphene was confirmed using X-ray photoelectron spectroscopy and their interaction with the graphene was analyzed from their core level spectra. Samples doped with $\mathrm{SOCl}_{2}$ showed the poorest temporal and thermal stability with both increasing time and temperature while samples doped with $\mathrm{HNO}_{3}$ performed better in both the scenarios. $\mathrm{MoO}_{3}$ doped samples showed the best stability with increasing time and temperature and seems to be the best candidate for applications in advanced electronics which require thermal stability. Our theory modeling results indicate the adsorption configurations of the three molecules are intact $\left(\mathrm{SOCl}_{2}\right)$, dissociated $\left(\mathrm{NO}_{2}\right)$ and clustered $\left(\mathrm{MoO}_{3}\right)_{\mathrm{n}}$. The p-type doping efficiencies are positively related to the resistance reduction in experiments and we have provided an interpretation of why annealing is essential when we seek to use $\mathrm{MoO}_{3}$ as a (stable) p-type dopant for graphene. The results could be very useful for graphene based interconnects and transparent electrodes where the resistance is a key factor.

Acknowledgements This work was supported by the Swedish Foundation for Strategic Research (SSF) Frame Project "Carbon Based 3D High Speed GaN Electronics Systems" (SE13-0061), The Production Area of Advance program, Chalmers University of Technology, Sweden. We also thank for the financial support by the Key R\&D Development Program from the Ministry of Science and Technology of China (2017YFB040600), the Chinese National Natural Science Foundation Project (U1537104) as well as Shanghai Education Commission Program (Shanghai University Peak Discipline Construction Project). Y. J and $\mathrm{P}$. $\mathrm{H}$ also acknowledge VR, Chalmers AoA Materials and computer time allocations by the Swedish National Infrastructure for Computing at NSC (Linkjöping) and C3SE (Gothenburg) for their support.

Open Access This article is distributed under the terms of the Creative Commons Attribution 4.0 International License (http://creativeco mmons.org/licenses/by/4.0/), which permits unrestricted use, distribution, and reproduction in any medium, provided you give appropriate credit to the original author(s) and the source, provide a link to the Creative Commons license, and indicate if changes were made.

\section{References}

1. A.A. Balandin, S. Ghosh, W. Bao, I. Calizo, D. Teweldebrhan, F. Miao, C.N. Lau, Superior thermal conductivity of single-layer graphene. Nano Lett. 8, 902-907 (2008)

2. H. Lüth, Solid Surfaces, Interfaces and Thin Films. (Springer, Dordrecht, 2010)

3. C.Y. Fong, B. Klein, in Diamond: Electronic Properties and Applications. The Kluwer International Series in Engineering and Computer Science, ed. by L.S. Pan, D.R. Kania, Ch. 1 (Springer, New york, (1995), pp. 1-29

4. J. Ristein, Surface transfer doping of semiconductors. Science 313, 1057-1058 (2006)

5. K. Berland et al., van der Waals density functionals built upon the electron-gas tradition: facing the challenge of competing interactions. J. Chem. Phys. 140, 18A539 (2014). https://doi. org/10.1063/1.4871731

6. A. Bergvall, K. Berland, P. Hyldgaard, S. Kubatkin, T. Löfwander, Graphene nanogap for gate-tunable quantum-coherent single-molecule electronics. Phys. Rev. B 84, 155451 (2011)

7. K. Berland, P. Hyldgaard, Analysis of van der Waals density functional components: binding and corrugation of benzene and C60 on boron nitride and graphene. Phys. Rev. B 87, 205421 (2013)

8. D. Hee Shin et al., Annealing effects on the characteristics of $\mathrm{AuCl}_{3}$-doped graphene. J. Appl. Phys. 113, 064305 (2013)

9. K.C. Kwon, B.J. Kim, J.-L. Lee, S.Y. Kim, Effect of anions in $\mathrm{Au}$ complexes on doping and degradation of graphene. J. Mater. Chem. C 1, 2463-2469 (2013)

10. K. Ki Kang et al., Enhancing the conductivity of transparent graphene films via doping. Nanotechnology 21, 285205 (2010)

11. K.C. Kwon, K.S. Choi, S.Y. Kim, Increased work function in few-layer graphene sheets via metal chloride doping. Adv. Func. Mater. 22, 4724-4731 (2012)

12. T. Takahashi, H. Tokailin, T. Sagawa, Angle-resolved ultraviolet photoelectron spectroscopy of the unoccupied band structure of graphite. Phys. Rev. B 32, 8317-8324 (1985)

13. S.M. Kim et al., Transparent organic P-dopant in carbon nanotubes: bis(trifluoromethanesulfonyl)imide. ACS Nano 4, 6998 7004 (2010)

14. S. Tongay et al., Stable hole doping of graphene for low electrical resistance and high optical transparency. Nanotechnology 22, 425701 (2011)

15. P. Wei et al., Tuning the Dirac point in CVD-grown graphene through solution processed n-type doping with 2-(2-methoxyphenyl)-1,3-dimethyl-2,3-dihydro-1H-benzoimidazole. Nano Lett. 13, 1890-1897 (2013)

16. X. Dong et al., Doping single-layer graphene with aromatic molecules. Small 5, 1422-1426 (2009)

17. I.-Y. Lee et al., Hydrazine-based n-type doping process to modulate Dirac point of graphene and its application to complementary inverter. Org. Electron. 14, 1586-1590 (2013)

18. W. Zhao, P. Tan, J. Zhang, J. Liu, Charge transfer and optical phonon mixing in few-layer graphene chemically doped with sulfuric acid. Phys. Rev. B 82, 245423 (2010)

19. A.C. Nicolás, A.A. Julio, The interaction of sulfuric acid with graphene and formation of adsorbed crystals. Nanotechnology 18, 485705 (2007)

20. V.M. Gun'ko et al., Interactions of single and multi-layer graphene oxides with water, methane, organic solvents and $\mathrm{HCl}$ studied by 1H NMR. Carbon 57, 191-201 (2013)

21. E. Bouleghlimat et al., The effect of acid treatment on the surface chemistry and topography of graphite. Carbon 61, 124-133 (2013) 
22. A. Kasry, M.A. Kuroda, G.J. Martyna, G.S. Tulevski, A.A. Bol, Chemical doping of large area stacked graphene films for use as transparent, conducting electrodes. ACS Nano 4, 3839-3844 (2010)

23. F. Fillaux et al., Inelastic neutron scattering study of the proton dynamics in $\mathrm{HNO} 3$ graphite intercalation compounds. Chem. Phys. 242, 273-281 (1999)

24. S. Das et al., Effect of HNO3 functionalization on large scale graphene for enhanced tri-iodide reduction in dye-sensitized solar cells. J. Mater. Chem. 22, 20490-20497 (2012)

25. S. Bae et al., Roll-to-roll production of 30-inch graphene films for transparent electrodes. Nat Nano 5, 574-578 (2010)

26. G. Eda et al., Transparent and conducting electrodes for organic electronics from reduced graphene oxide. Appl. Phys. Lett. 92, 233305 (2008)

27. X. Li et al., in Proceedings of the Conference on China Technological Development of Renewable Energy Source, vol. I (China, 2010), pp. 387-390

28. X. Li et al., Ion doping of graphene for high-efficiency heterojunction solar cells. Nanoscale 5, 1945-1948 (2013)

29. T. Cui et al., Enhanced efficiency of graphene/silicon heterojunction solar cells by molecular doping. J. Mater. Chem. A 1, 5736-5740 (2013)

30. Z. Chen et al., Surface transfer hole doping of epitaxial graphene using MoO3 thin film. Appl. Phys. Lett. 96, 213104 (2010)

31. L. Xie et al., Electrical measurement of non-destructively p-type doped graphene using molybdenum trioxide. Appl. Phys. Lett. 99, $012112(2011)$

32. S.L. Hellstrom, M. Vosgueritchian, R.M. Stoltenberg, I. Irfan, M. Hammock, Y.B. Wang, C. Jia, X. Guo, Y. Gao, Z. Bao, Strong and stable doping of carbon nanotubes and graphene by MoOx for transparent electrodes. Nano Lett. 12, 3574-3580 (2012)

33. J. Meyer, et al, Metal oxide induced charge transfer doping and band alignment of graphene electrodes for efficient organic light emitting diodes. Sci. Rep. 4, 5380 (2014). https://doi.org/10.1038/ srep05380

34. Y. Guo, J. Robertson, Origin of the high work function and high conductivity of MoO3. Appl. Phys. Lett. 105, 222110 (2014). https://doi.org/10.1063/1.4903538

35. P.R. Kidambi, C. Weijtens, J. Robertson, S. Hofmann, J. Meyer, Multifunctional oxides for integrated manufacturing of efficient graphene electrodes for organic electronics. Appl. Phys. Lett. 106, 063304 (2015). https://doi.org/10.1063/1.4908292
36. S. Sanders et al., Engineering high charge transfer n-doping of graphene electrodes and its application to organic electronics. Nanoscale 7, 13135-13142 (2015). https://doi.org/10.1039/ C5NR03246F

37. L.G. Gerling et al., Transition metal oxides as hole-selective contacts in silicon heterojunctions solar cells. Sol. Energy Mater. Sol. Cells (2016). https://doi.org/10.1016/j.solmat.2015.08.028

38. Q. Cao et al., End-bonded contacts for carbon nanotube transistors with low, size-independent resistance. Science 350, 68-72 (2015)

39. Q.-H. Wu et al., Electronic structure of $\mathrm{MoO} 3-\mathrm{x} /$ graphene interface. Carbon 65, 46-52 (2013)

40. S. Esconjauregui, et al, Efficient transfer doping of carbon nanotube forests by MoO3. ACS Nano 9, 10422-10430 (2015)

41. M. Dion, H. Rydberg, E. Schröder, D.C. Langreth, B.I. Lundqvist, Van der Waals density functional for general geometries. Phys. Rev. Lett. 92, 246401 (2004)

42. M. Dion, H. Rydberg, E. Schröder, D.C. Langreth, B.I. Lundqvist, Erratum: Van der Waals density functional for general geometries. [Phys. Rev. Lett. 92, 246401 (2004)]. Phys. Rev. Lett. 95, 109902 (2005)

43. T. Thonhauser et al., Spin signature of nonlocal correlation binding in metal-organic frameworks. Phys. Rev. Lett. 115, 136402 (2015)

44. K. Berland, P. Hyldgaard, Exchange functional that tests the robustness of the plasmon description of the van der Waals density functional. Phys. Rev. B 89, 035412 (2014)

45. G. Paolo et al., QUANTUM ESPRESSO: a modular and opensource software project for quantum simulations of materials. J. Phys. 21, 395502 (2009)

46. G. Henkelman, A. Arnaldsson, H. Jónsson, A fast and robust algorithm for Bader decomposition of charge density. Comput. Mater. Sci. 36, 354-360 (2006). https://doi.org/10.1016/j.comma tsci.2005.04.010

47. R. Car, M. Parrinello, Unified approach for molecular dynamics and density-functional theory. Phys. Rev. Lett. 55, 2471 (1985)

48. S. HJ et al., Tailoring electronic structures of carbon nanotubes by solvent with electron-donating and -withdrawing groups. J. Am. Chem. Soc. 13, 2062-2066 (2008)

49. D. Le, A. Kara, E. Schröder, P. Hyldgaard, T.S. Rahman, Physisorption of nucleobases on graphene: a comparative van der Waals study. J. Phys. 24, 424210 (2012) 